\title{
Um limitante superior para \\ a probabilidade crítica do modelo dos sapos em árvores homogêneas
}

\section{Élcio Lebensztayn}

\author{
TESE APRESENTADA \\ AO INSTITUTO DE MATEMÁTICA E ESTATÍSTICA \\ DA UNIVERSIDADE DE SÃO PAULO \\ PARA OBTENÇÃO \\ DO GRAU DE DOUTOR EM ESTATÍSTICA
}

\author{
Área de Concentração: Probabilidade \\ Orientadores: Prof. Dr. Fábio Prates Machado \\ Prof. Dr. Serguei Popov
}

São Paulo, 18 de agosto de 2005.

O autor agradece à FAPESP pelo apoio financeiro (Processo N. 01/04416-0). 


\section{Um limitante superior para \\ a probabilidade crítica do modelo dos sapos em árvores homogêneas}

Este exemplar corresponde à tese defendida por Élcio Lebensztayn e aprovada pela comissão julgadora.

São Paulo, 18 de agosto de 2005.

Banca examinadora:

Prof. Dr. Fábio Prates Machado (IME-USP)

Prof. Dr. Luiz Renato Gonçalves Fontes (IME-USP)

Profa. Dra. Marina Vachkovskaia (UNICAMP)

Profa. Dra. Nancy Lopes Garcia (UNICAMP)

Prof. Dr. Oswaldo Scarpa Magalhães Alves (UFG) 
Para Frida, Jacob e Ieda. 


\section{Agradecimentos}

Agradeço aos professores com os quais convivi nesses anos de formação acadêmica, em especial aos meus orientadores Prof. Dr. Fábio Prates Machado e Prof. Dr. Serguei Popov. Também aos amigos da pós-graduação, em particular a Mauricio Zuluaga Martinez.

Agradeço aos meus pais Frida e Jacob e à minha irmã Ieda.

À FAPESP, pelo apoio recebido.

A Deus e a todos que me ajudaram a chegar até aqui. 


\section{Resumo}

Estudamos o modelo dos sapos na árvore homogênea, um sistema de partículas a tempo discreto cuja dinâmica é sintetizada a seguir. No instante inicial, existe em cada vértice da árvore um número aleatório independente e identicamente distribuído de partículas; aquelas posicionadas em um vértice fixado estão ativas, as demais inativas. Partículas ativas realizam passeios aleatórios simples, independentes, a tempo discreto, com probabilidade de desaparecimento $(1-p)$ em cada instante. Uma partícula inativa torna-se ativa assim que seu vértice é visitado por uma partícula ativa. Consideramos nesta tese o valor crítico $p_{c}$ que separa a fase em que o processo se extingue quase certamente da fase em que existem partículas ativas em todos os instantes com probabilidade positiva. Provamos um limitante superior para a probabilidade crítica $p_{c}$, o qual melhora o resultado anteriormente conhecido para o caso de configuração inicial de uma partícula por vértice. O argumento utilizado consiste na descrição do modelo dos sapos como um modelo de percolação orientada que domina processos de ramificação convenientemente definidos. Obtemos também o valor assintótico do limitante superior estabelecido, mostrando ser igual ao valor assintótico da probabilidade crítica.

Palavras-chave: modelo dos sapos, probabilidade crítica, árvore homogênea, percolação orientada. 


\section{Abstract}

We study the frog model on the homogeneous tree, a discrete-time particle system whose dynamics is summarized next. Initially there is an independent and identically distributed random number of particles at each vertex of the tree; those placed at a fixed vertex are active, the others being inactive. Active particles perform independent discrete-time simple random walks, with probability of disappearance $(1-p)$ at each instant. An inactive particle becomes active once its vertex is hit by an active particle. We consider in this thesis the critical value $p_{c}$ that separates the phase in which the process dies out almost surely from the phase in which there exist active particles at all times with positive probability. We prove an upper bound for the critical probability $p_{c}$, which improves the formerly known result for the case of one particle per vertex initial configuration. The employed argument builds on the description of the frog model as an oriented percolation model which dominates suitably defined branching processes. We also obtain the asymptotic value of the stated upper bound, showing that it equals the asymptotic value of the critical probability.

Key words: frog model, critical probability, homogeneous tree, oriented percolation. 


\section{Sumário}

Notações

1 Modelo dos sapos 2

1.1 Introdução . . . . . . . . . . . . . . . . . . . 2

1.2 Definições . . . . . . . . . . . . . . . . . . . . . 4

1.3 Referências sobre o modelo . . . . . . . . . . . . . . . 9 9

1.4 Organização da tese . . . . . . . . . . . . . . . . 10

$\begin{array}{lll}2 & \text { Limitante superior para a probabilidade crítica } & 11\end{array}$

2.1 Resultados principais . . . . . . . . . . . . . . . . . 11

$2.2 \operatorname{MS}\left(\mathbb{T}_{d}, p, \eta\right)$ como um modelo de percolação $\ldots \ldots \ldots$

2.3 Processos de ramificação dominados por $\operatorname{MS}\left(\mathbb{T}_{d}, p, \eta\right) \ldots \ldots$

2.3.1 Provas dos lemas .................... 20

2.4 Observações . . . . . . . . . . . . . . . . . . . . . . . 23

3 Correção assintótica $\quad 25$

3.1 Limitante inferior para a probabilidade crítica . . . . . . . . 25

3.2 Valor assintótico . . . . . . . . . . . . . . . 27

3.3 Prova do teorema $3.1 \ldots \ldots \ldots \ldots \ldots$

$\begin{array}{lll}\text { Apêndice } & \text { Convergência uniforme } & 30\end{array}$

$\begin{array}{ll}\text { Referências Bibliográficas } & 33\end{array}$ 


\section{Lista de Figuras}

1.1 Uma representação da árvore homogênea de grau $3 \quad \ldots$. . . . . . . . 4

1.2 Os primeiros instantes de uma realização do modelo dos sapos em $\mathbb{T}_{2}$ com configuração inicial de uma partícula por vértice . . . . . . . 8

2.1 Em $\mathbb{T}_{2}$, uma representação do caminho entre a raiz e um vértice $x$ e do conjunto $\mathbb{T}_{2}^{+}(x) \ldots \ldots \ldots \ldots \ldots \ldots$ 


\section{Notações}

Conjuntos. $\mathbb{N}=\{0,1,2, \ldots\}$ é o conjunto dos números naturais;

$\mathbb{Z}=\{\ldots,-2,-1,0,1,2, \ldots\}$ é o conjunto dos números inteiros;

$\mathbb{R}$ é o conjunto dos números reais.

Grafos. Com o abuso usual de notação, para $d=1,2, \ldots$, denotamos $\mathbb{Z}^{d}$ a rede hipercúbica $d$-dimensional, isto é, o grafo com conjunto de vértices $\left\{\left(x^{(1)}, \ldots, x^{(d)}\right)\right.$ : $\left.x^{(i)} \in \mathbb{Z}, i=1, \ldots, d\right\}$ e em que dois vértices são ligados por um elo se estão à distância euclidiana 1.

Para $d=2,3, \ldots$, denotamos $\mathbb{T}_{d}$ a árvore homogênea de grau $(d+1)$.

Terminologia. Uma função $f: X \rightarrow \mathbb{R}$ (onde $X \subset \mathbb{R}$ ) é chamada crescente se, quaisquer que sejam $x, y \in X, x<y$ implica $f(x)<f(y)$. Se $x<y(\operatorname{com} x, y \in X)$ implica apenas $f(x) \leq f(y), f$ é dita não-decrescente. De modo análogo, define-se função decrescente e função não-crescente. Uma função de qualquer destes tipos é chamada monótona.

Definições análogas são consideradas para uma seqüência $\left\{x_{n}\right\}$ de números reais. Assim, por exemplo, a seqüência $\left\{x_{n}\right\}$ de números reais é não-crescente se $x_{n+1} \leq x_{n}$ para todo $n$. 


\section{Capítulo 1}

\section{Modelo dos sapos}

\subsection{Introdução}

Probabilidade em árvores é uma área rica e de vigoroso desenvolvimento recente, que tem atraído o interesse de muitos probabilistas. De forma geral, é a ausência de circuitos que torna os cálculos mais simples, e mais pode ser feito para processos em árvores do que em grafos gerais. Para o modelo de percolação independente na árvore binária, por exemplo, é possível obter exatamente a probabilidade crítica e os expoentes críticos; além disso, este é um caso em que se pode apreender o comportamento nas redes hipercúbicas de alta dimensão a partir do estudo na árvore (veja-se o capítulo 10 de Grimmett [8]). Diferentemente, o principal motivo do interesse no processo de contato nas árvores homogêneas de grau maior que 2 foi o fato de existir uma transição de fase múltipla neste contexto, o que não ocorre no caso das redes hipercúbicas (mais detalhes encontram-se na parte I de Liggett [11]). Sobre a teoria de certos processos probabilísticos em grafos infinitos, especialmente em árvores, sugerimos o livro de Lyons e Peres [13].

O assunto desta tese é o modelo dos sapos, um sistema de partículas cujos agentes (passeios aleatórios com tempo de vida aleatório) se movem entre os vértices de um grafo, ao longo dos elos. Em resumo, visamos a estudá-lo na árvore homogênea, mostrando um limitante superior para a probabilidade crítica de sobrevivência do modelo.

Detalhando o modelo estudado, as partículas movem-se como passeios aleatórios simples a tempo discreto, independentes, nos vértices de um grafo $\mathcal{G}$, morrendo após 
um tempo de vida aleatório com distribuição geométrica. Inicialmente, existe em cada vértice de $\mathcal{G}$ um número aleatório independente de partículas; na forma do modelo mais comumente estudada, este número é identicamente distribuído. No tempo zero, todas as partículas estão inativas, exceto aquelas porventura posicionadas em um vértice fixado do grafo, denominado raiz. Em cada instante de tempo, cada partícula ativa morre com probabilidade $(1-p)$ ou sobrevive com probabilidade $p$, independentemente das demais. Uma vez que sobrevive, salta para um dos vértices vizinhos mais próximos, escolhido com probabilidade uniforme, realizando um passeio aleatório simples a tempo discreto em $\mathcal{G}$. Até o tempo em que morre, ela ativa todas as partículas inativas que encontra pelo caminho. A partir do momento em que é ativada, cada partícula começa a passear, realizando exatamente a mesma dinâmica, independentemente de qualquer coisa.

O modelo dos sapos é uma modificação de um modelo para a difusão de uma informação numa rede que foi proposto por K. Ravishankar. A fim de entender esta interpretação, imagine-se que cada partícula ativa carrega uma informação que compartilha com todas as partículas inativas que encontra durante sua vida. Uma vez que a informação é passada para uma partícula inativa, esta é ativada e começa a se mover, ajudando a espalhar a informação. O modelo com $p=1$ (ou seja, sem morte) é uma versão a tempo discreto do modelo proposto em 1996 por R. Durrett (comunicação pessoal com F. P. Machado), que sugeriu o nome frog model. A versão com morte foi formulada por I. Benjamini em 2000. Na seção 1.3, apresentamos uma síntese sobre as referências acerca do modelo.

Destacam-se para o modelo dos sapos dois comportamentos críticos fundamentais com respeito ao parâmetro $p$ : um relativo à sobrevivência (existência ad ceternum de partículas ativas) e outro com relação à recorrência (infinidade de visitas de partículas ativas à raiz). Neste trabalho, a probabilidade crítica considerada é com respeito à sobrevivência do modelo. Reiteramos que nosso objetivo primordial é estabelecer um limitante superior para a probabilidade crítica do modelo na árvore homogênea. 


\subsection{Definições}

Em geral, o modelo dos sapos é definido sobre um grafo $\mathcal{G}$ infinito, conectado e localmente finito. Na tese, consideramos $\mathcal{G}=\mathbb{T}_{d}=(\mathcal{V}, \mathcal{E})$ a árvore homogênea de grau $(d+1), d \in\{2,3, \ldots\}$, que é o (único) grafo conectado, desprovido de circuitos, cujos vértices têm todos grau $(d+1)$. Mais detalhes sobre Teoria de Grafos podem ser encontrados, por exemplo, em Woess [18]; citamos ainda Bollobás [5] para quem se interesse especialmente por esse assunto. Uma parte de $\mathbb{T}_{2}$ é ilustrada na figura 1.1. Na notação precedente, $\mathcal{V}$ e $\mathcal{E}$ são respectivamente o conjunto de vértices e o conjunto de elos de $\mathbb{T}_{d}$. Dizemos que $x, y \in \mathcal{V}$ são vizinhos se pertencem a um elo comum, o que denotamos por $x \sim y$. Para cada par de vértices $x, y \in \mathcal{V}$, existe um único caminho conectando $x$ e $y$, isto é, uma única seqüência $x=x_{0}, x_{1}, \ldots, x_{n}=y$ de vértices distintos na qual $x_{i} \sim x_{i+1}$ para cada $i=0, \ldots, n-1$. O valor $n$ na última frase é a distância entre $x$ e $y$, denotada dist $(x, y)$. Um vértice $\mathbf{0}$ de $\mathbb{T}_{d}$ é fixado e denominado a raiz de $\mathbb{T}_{d}$.

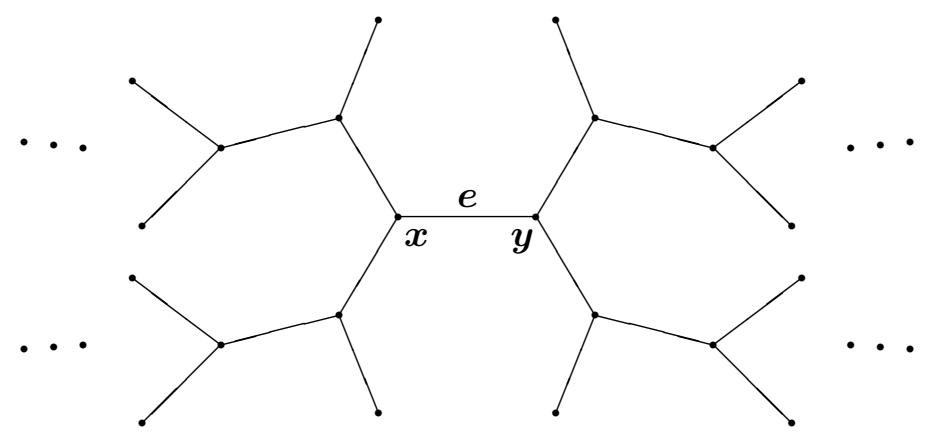

Figura 1.1: Uma representação de $\mathbb{T}_{2}$, a árvore homogênea de grau 3.

Ora definimos formalmente o modelo dos sapos em $\mathbb{T}_{d}$. Os símbolos $\mathbf{P}$ e $\mathbf{E}$ denotam a medida de probabilidade e a esperança associada, respectivamente. Seja $\eta$ uma variável aleatória assumindo valores em $\mathbb{N}$, tal que $\mathbf{P}(\eta \geq 1)>0$. Definimos $p_{j}=\mathbf{P}(\eta=j), j \in \mathbb{N}, m=\mathbf{E} \eta$ e $\varphi$ a função geradora de probabilidade de $\eta$. Sejam 
$\{\eta(x) ; x \in \mathcal{V}\},\left\{\left(S_{n}^{x}(i)\right)_{n \in \mathbb{N}} ; i \in\{1,2,3, \ldots\}, x \in \mathcal{V}\right\}$ e $\left\{\Xi_{p}^{x}(i) ; i \in\{1,2,3, \ldots\}, x \in \mathcal{V}\right\}$ conjuntos independentes de objetos aleatórios independentes e identicamente distribuídos, descritos a seguir. Para cada $x \in \mathcal{V}, \eta(x)$ tem a mesma distribuição de $\eta$ e fornece o número inicial de partículas no vértice $x$. Se $\eta(x) \geq 1$, então, para cada $1 \leq i \leq \eta(x),\left(S_{n}^{x}(i)\right)_{n \in \mathbb{N}}$ é um passeio aleatório simples a tempo discreto em $\mathbb{T}_{d}$ começando em $x$, e $\Xi_{p}^{x}(i)$ é uma variável aleatória com distribuição dada por $\mathbf{P}\left(\Xi_{p}^{x}(i)=k\right)=(1-p) p^{k-1}, k=1,2, \ldots$, onde $p \in[0,1]$ é um parâmetro fixado. Fica definido dessa forma o destino aleatório (trajetória e tempo de vida) de cada uma das $\eta(x)$ partículas posicionadas originalmente em $x$, as quais começam a se mover no instante em que são ativadas (caso isto venha a ocorrer). Quando ativada, a $i$-ésima partícula em $x$ segue o passeio aleatório $\left(S_{n}^{x}(i)\right)_{n \in \mathbb{N}}$ e morre (desaparece) $\Xi_{p}^{x}(i)$ unidades de tempo após ser ativada. No tempo zero, estão ativas apenas as partículas que tenham sido posicionadas em $\mathbf{0}$.

É essencial reparar que, em cada instante de tempo, uma partícula ativa primeiro decide se sobrevive ou não, e então, no caso de sobrevivência, salta. Isto significa que o número de saltos dados pela $i$-ésima partícula em $x$, quando ativada, é igual a $\Xi_{p}^{x}(i)-1$. Note-se também que não há interação entre partículas ativas: cada partícula ativa se move independentemente de qualquer coisa. O modelo recém-definido é conhecido como modelo dos sapos em $\mathbb{T}_{d}$, com parâmetro de sobrevivência $p$ e configuração inicial dada por cópias independentes de $\eta$ em cada vértice de $\mathbb{T}_{d}$, o qual denotamos por $\operatorname{MS}\left(\mathbb{T}_{d}, p, \eta\right)$.

A figura 1.2 (p. 8) ilustra os primeiros instantes de uma possível realização do modelo dos sapos em $\mathbb{T}_{2}$, com configuração inicial de uma partícula por vértice (ou seja, $\eta \equiv 1$ ). Círculos claros e escuros representam respectivamente partículas inativas e ativas. Descrevemos o que ocorre:

- No tempo $n=0$, somente a partícula da raiz está ativa.

- Esta partícula decide sobreviver, após o que escolhe saltar para o vértice nomeado y, 
ativando a partícula deste vértice.

- No tempo $n=1$, existem duas partículas em $y$, ambas ativas, a raiz está vazia e cada um dos demais vértices possui exatamente uma partícula inativa.

- Ambas as partículas de y sobrevivem, uma salta para a raiz e a outra para o vértice $z$, ativando a partícula aí posicionada.

- Tem-se a seguinte configuração quando $n=2$ : o vértice $y$ vazio, uma partícula ativa em $\mathbf{0}$, duas partículas ativas em $z$ e cada um dos demais vértices com uma partícula inativa.

- A partícula em 0 sobrevive e salta para o vértice $x$; uma das partículas em $z$ morre e a outra sobrevive e salta para o vértice $w$.

- Quando $n=3$, existem duas partículas ativas em $x$, assim como em $w$; os vértices $\mathbf{0}$, $y$ e $z$ estão vazios e cada um dos demais vértices tem uma partícula inativa.

Para introduzir a criticalidade, necessitamos da seguinte definição.

Definição 1.1. Uma realização particular do modelo dos sapos sobrevive se, para todo instante de tempo, existe pelo menos uma partícula ativa. Caso contrário, dizemos que a realização se extingue.

Observamos então que $\mathbf{P}\left(\mathrm{MS}\left(\mathbb{T}_{d}, p, \eta\right)\right.$ sobrevive $)$ é não-decrescente em $p$, logo definimos a probabilidade crítica do modelo dos sapos em $\mathbb{T}_{d}$

$$
p_{c}\left(\mathbb{T}_{d}, \eta\right)=\inf \left\{p: \mathbf{P}\left(\mathrm{MS}\left(\mathbb{T}_{d}, p, \eta\right) \text { sobrevive }\right)>0\right\}
$$

No caso particular de $\eta \equiv 1$ (configuração inicial de uma partícula por vértice), simplificamos a notação da probabilidade crítica para $p_{c}\left(\mathbb{T}_{d}\right)$.

Conforme demonstrado em Alves et al. [2], sob a já suposta condição de que $\mathbf{P}(\eta \geq 1)>0$, temos que $p_{c}\left(\mathbb{T}_{d}, \eta\right)<1$ para todo $d \geq 2$, isto é, o modelo dos sapos em $\mathbb{T}_{d}$ sobrevive com probabilidade positiva para $p$ suficientemente perto de 1 . Nesse artigo também se prova que a probabilidade crítica $p_{c}\left(\mathbb{T}_{d}, \eta\right)$ é positiva se e somente se 
existe $\delta>0$ tal que $\mathbf{E} \eta^{\delta}<\infty$. Assim, a última condição é necessária e suficiente para que $\operatorname{MS}\left(\mathbb{T}_{d}, p, \eta\right)$ exiba transição de fase, ou seja,

$$
0<p_{c}\left(\mathbb{T}_{d}, \eta\right)<1
$$

Em particular, fica demonstrada a transição de fase no caso em que $\eta \equiv 1$.

Um limitante superior explícito para a probabilidade crítica era conhecido somente no caso de configuração inicial de uma partícula por vértice, a saber, $p_{c}\left(\mathbb{T}_{d}\right) \leq$ $(d+1)(2 d-2)^{-1}$ para $d \geq 4$ (Fontes et al. [7]). Estabelecemos na tese um limitante superior no caso de configuração inicial aleatória, o qual, como subproduto, melhora o referido resultado quando a configuração inicial é de uma partícula por vértice. 
Tempo $n=0$ :

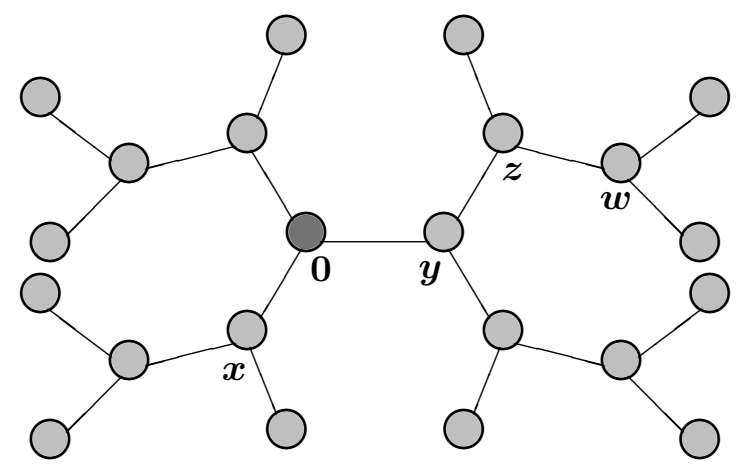

Tempo $n=1$ :

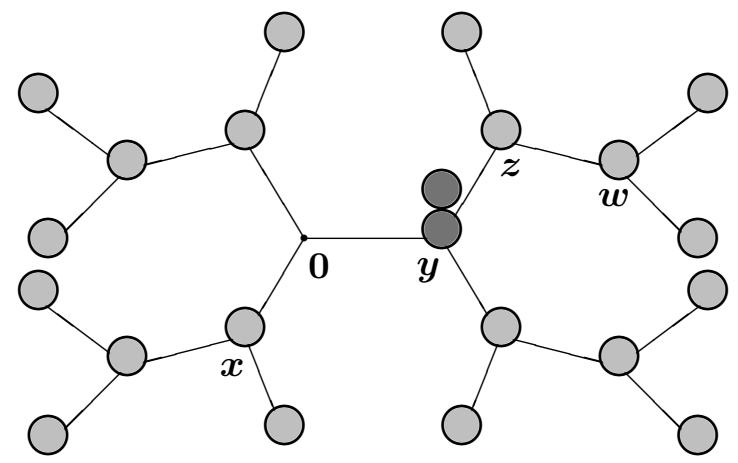

Tempo $n=2$ :

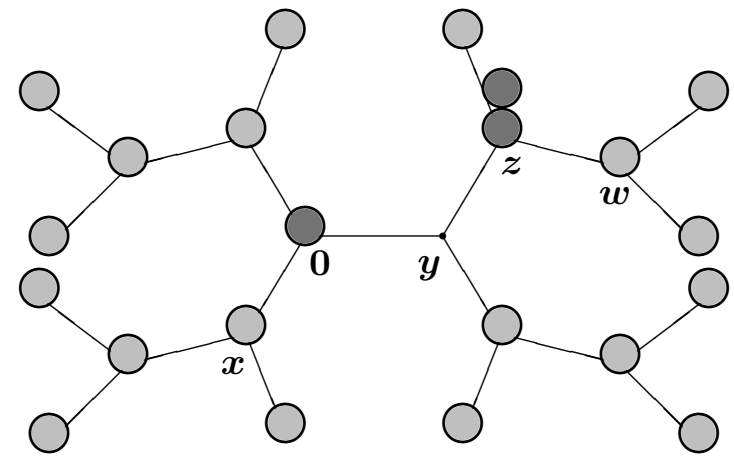

Tempo $n=3$ :

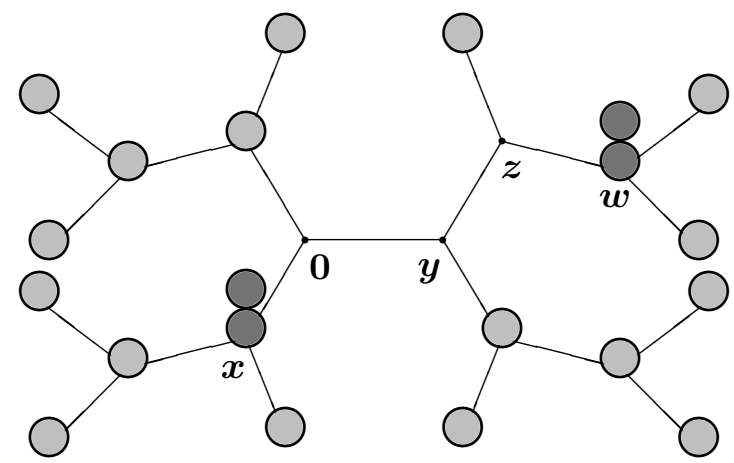

Figura 1.2: Os primeiros instantes de uma realização do modelo dos sapos em $\mathbb{T}_{2}$ com $\eta \equiv 1$. (Veja-se a explicação no texto). 


\subsection{Referências sobre o modelo}

Ao que se saiba, é de autoria de Telcs e Wormald [17] o primeiro artigo publicado lidando com o modelo dos sapos, que é chamado egg model. Provam que o modelo com $p=1, \mathcal{G}=\mathbb{Z}^{d}$ e configuração inicial de uma partícula por vértice é recorrente, ou seja, a raiz é visitada infinitas vezes quase certamente. Em [15], Popov considera o modelo com $p=1, \mathcal{G}=\mathbb{Z}^{d}(d \geq 3)$ e configuração inicial com densidade $p(x)$, identificando a taxa crítica de decaimento de $p(x)$ que separa a transitoriedade da recorrência.

Para o modelo dos sapos com $p=1, \mathcal{G}=\mathbb{Z}^{d}$ e configuração inicial de uma partícula por vértice, Alves et al. [3] estabelecem um resultado conhecido como teorema da forma. Em palavras, demonstram que o conjunto das posições originais de todas as partículas ativas, reescalado pelo tempo decorrido, converge para um conjunto compacto, convexo, não-vazio. Um resultado semelhante no caso de configuração inicial aleatória é obtido em Alves et al. [4]. Ramírez e Sidoravicius [16] consideram uma variante a tempo contínuo do modelo dos sapos sem mortes em $\mathbb{Z}^{d}$, com configuração inicial de uma partícula por vértice, provando o teorema da forma (por um método diferente de $[3,4])$, assim como o fato de que a distribuição local de partículas tende ao produto de Poissons.

Em Alves et al. [2], encontram-se resultados sobre transição de fase e valores assintóticos para a probabilidade crítica do modelo em $\mathbb{Z}^{d}$ e $\mathbb{T}_{d}$. Popov [14] apresenta um resumo dos resultados conhecidos e algumas questões em aberto, e Fontes et al. [7] mostram que, em geral, a probabilidade crítica não é uma função monótona do grafo. O resultado central desta tese é apresentado em Lebensztayn et al. [9].

Cumpre também mencionar os dois trabalhos de pós-graduação desenvolvidos sobre o modelo dos sapos. Na tese de Alves [1], em que é chamado modelo de reação em cadeia, são provados o teorema da forma para o modelo com $p=1$ e $\mathcal{G}=\mathbb{Z}^{d}$, assim como resultados a respeito de transição de fase em $\mathbb{Z}^{d}$ e $\mathbb{T}_{d}$. A dissertação de Leichsenring [10] aborda a não-monotonicidade da probabilidade crítica como função do grafo. 


\subsection{Organização da tese}

No próximo capítulo, apresentamos e demonstramos o resultado principal da tese, que estabelece um limitante superior para a probabilidade crítica do modelo dos sapos em $\mathbb{T}_{d}$. O capítulo 3 é dedicado à prova da correção assintótica deste limitante, isto é, a mostrar que o limitante converge quando $d \rightarrow \infty$ para o valor assintótico da probabilidade crítica. Para esclarecer certas passagens das demonstrações, finalizamos a tese com um apêndice sobre convergência uniforme.

Convém ressaltar que, em linhas gerais, foram mantidas as notações adotadas em Lebensztayn et al. [9]. Uma diferença marcante entre a tese e o artigo é que optamos na tese por tratar diretamente do caso de configuração inicial aleatória; no artigo, o caso de configuração inicial de uma partícula por vértice é abordado separadamente e são omitidos os detalhes da demonstração no caso aleatório. A discussão sobre a correção assintótica do limitante não está incluída no artigo. 


\section{Capítulo 2}

\section{Limitante superior para a probabilidade crítica}

\subsection{Resultados principais}

O teorema central da tese estabelece um limitante superior estritamente menor que 1 para a probabilidade crítica do modelo dos sapos na árvore homogênea de grau $(d+1), d \geq 2$, em que a configuração inicial de partículas é dada por cópias independentes de $\eta$ em cada vértice do grafo. Recordamos que $\eta$ é uma variável aleatória inteira e não-negativa, $\operatorname{com} p_{0}=\mathbf{P}(\eta=0)<1$. Além disso,

$$
\varphi(s)=\sum_{k=0}^{\infty} p_{k} s^{k}, s \in[0,1]
$$

é a função geradora de probabilidade de $\eta$.

Teorema 2.1. Para todo $d \geq 2$ fixado,

$$
p_{c}\left(\mathbb{T}_{d}, \eta\right) \leq \frac{\gamma(d+1)[d(\alpha+\gamma)-\sqrt{\Delta}]}{d\{(\alpha+\gamma)[d(\alpha+\gamma)-\sqrt{\Delta}]-2 \gamma(1-\gamma)\}}<1,
$$

onde

$$
\begin{gathered}
\theta=\varphi(1-1 / d), \\
\alpha=p_{0}+d(1-\theta), \\
\gamma=\frac{d}{d-1}\left(\theta-p_{0}\right), \\
\Delta=d\left[-4 \gamma+d(\alpha+\gamma)^{2}\right] .
\end{gathered}
$$


Lembrando que $p_{c}\left(\mathbb{T}_{d}\right)$ denota a probabilidade crítica no caso em que $\eta \equiv 1$, cumpre destacar o seguinte corolário do teorema 2.1.

Teorema 2.2. Para todo $d \geq 2$ fixado,

$$
p_{c}\left(\mathbb{T}_{d}\right) \leq \frac{d+1}{2 d}
$$

Enfatizamos que este limitante superior para $p_{c}\left(\mathbb{T}_{d}\right)$ é melhor que o anteriormente conhecido (a saber, $(d+1)(2 d-2)^{-1}, d \geq 4$, apresentado em Fontes et al. [7]).

Dedicamo-nos nas duas próximas seções à prova do teorema 2.1. Durante toda a demonstração, consideramos $d \geq 2$ fixado. Em resumo, o argumento principal é a descrição do modelo dos sapos em $\mathbb{T}_{d}$ como um modelo de percolação orientada que domina processos de ramificação convenientemente definidos. Com a finalidade de clareza, a prova é separada nos dois passos sintetizados a seguir:

- Na seção 2.2, explicamos por que o modelo dos sapos pode ser visto como um modelo de percolação orientada e provamos uma fórmula para a probabilidade de um elo orientado estar aberto.

- Na seção 2.3, definimos para cada $n$ um processo de ramificação de GaltonWatson que é dominado pelo modelo dos sapos, isto é, tal que o modelo dos sapos sobrevive se este processo o fizer. Para cada um destes processos de ramificação, obtemos uma condição suficiente para comportamento supercrítico, o que nos fornece uma seqüência de limitantes superiores para $p_{c}\left(\mathbb{T}_{d}, \eta\right)$. Esta seqüência converge para o limitante superior dado no teorema 2.1. 


\section{$2.2 \mathrm{MS}\left(\mathbb{T}_{d}, p, \eta\right)$ como um modelo de percolação}

É essencial observar que estamos trabalhando com um modelo de percolação. Para esclarecer esta assertiva, consideremos a seguinte definição.

Definição 2.1. (i) Para $x \in \mathcal{V}$, seja

$$
\mathcal{R}_{x}^{i}=\left\{S_{n}^{x}(i): 0 \leq n<\Xi_{p}^{x}(i)\right\} \subset \mathcal{V}
$$

o conjunto virtual de vértices visitados pela $i$-ésima partícula posicionada originalmente em $x$. Definimos

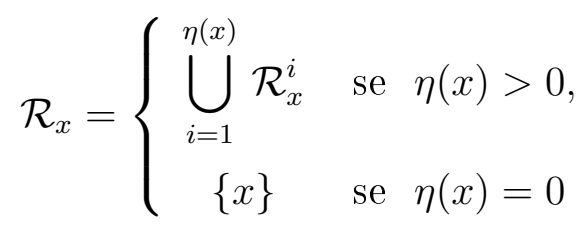

o traço virtual do vértice $x$.

(ii) Para $x, y \in \mathcal{V}$ distintos, definimos

$$
\{x \rightarrow y\}=\left\{y \in \mathcal{R}_{x}\right\} \quad \text { e } \quad\{x \nrightarrow y\}=\left\{y \notin \mathcal{R}_{x}\right\}
$$

Usamos a palavra 'virtual' porque não se sabe se as partículas do vértice $x$ são ou não ativadas. Assim, para $x, y \in \mathcal{V}$ distintos, $\{x \rightarrow y\}$ é o evento de que alguma partícula posicionada originalmente em $x$ visita $y$ virtualmente.

Seja $\overrightarrow{\mathbb{T}}_{d}$ o grafo orientado com conjunto de vértices $\mathcal{V}$ e um elo orientado de $x$ a $y$ para cada par $(x, y) \in \mathcal{V} \times \mathcal{V}$ tal que $x \neq y$. Tendo em vista a definição 2.1, concluímos que o modelo dos sapos em $\mathbb{T}_{d}$ pode ser visto como um modelo de percolação orientada, dependente, de longo alcance, em $\overrightarrow{\mathbb{T}}_{d}$. Ademais, a sobrevivência de $\operatorname{MS}\left(\mathbb{T}_{d}, p, \eta\right)$ é equivalente à existência de uma seqüência infinita de vértices distintos $\mathbf{0}=x_{0}, x_{1}, x_{2}, \ldots$, tal que $x_{j} \rightarrow x_{j+1}$ para todo $j \geq 0$.

Observação 2.1. Embora o modelo dos sapos seja um modelo particular de percolação, Fontes et al. [7] provam que a probabilidade crítica não é uma função monótona do grafo. Este é um fato um tanto inesperado, já que para os modelos usuais de percolação vale a monotonicidade no grafo da probabilidade crítica (consulte-se Grimmett [8]). 
Um aspecto de grande importância é que podemos determinar uma fórmula para a probabilidade do evento $\{x \rightarrow y\}$. Como veremos a seguir, contribui fundamentalmente para isto o fato de estarmos lidando com a árvore homogênea.

Proposição 2.1. Para $x, y \in \mathcal{V}$ distintos com $\operatorname{dist}(x, y)=n$, temos

$$
\mathbf{P}(x \rightarrow y)=1-\varphi\left(1-(B(p, d))^{n}\right),
$$

onde

$$
B(p, d)=\left\{\begin{array}{cl}
\frac{d+1-\sqrt{(d+1)^{2}-4 d p^{2}}}{2 d p} & \text { se } 0<p \leq 1 \\
0 & \text { se } p=0
\end{array}\right.
$$

Prova. Sejam $x, y \in \mathcal{V}$ distintos com $\operatorname{dist}(x, y)=n$ e consideremos $p>0$, já que o caso $p=0$ é trivial. Por condicionamento no número inicial de partículas em $x$, temos

$$
\mathbf{P}(x \rightarrow y)=1-\varphi\left(\mathbf{P}\left(y \notin \mathcal{R}_{x}^{1}\right)\right)
$$

logo nos resta provar que

$$
\mathbf{P}\left(y \in \mathcal{R}_{x}^{1}\right)=\left[\frac{d+1-\sqrt{(d+1)^{2}-4 d p^{2}}}{2 d p}\right]^{n} .
$$

Seja $T_{x y}$ o primeiro tempo em que o passeio aleatório $\left(S_{n}^{x}(1)\right)_{n \in \mathbb{N}}$ visita o vértice $y$. Para $p<1$, temos

$$
\mathbf{P}\left(y \in \mathcal{R}_{x}^{1}\right)=\sum_{\ell=n}^{\infty} \mathbf{P}\left(\Xi_{p}^{x}(1)>\ell, T_{x y}=\ell\right)=\mathbf{E}\left(p^{T_{x y}}\right) .
$$

Visto que $T_{x y}$ é uma soma de $n$ cópias independentes de $T_{x x^{\prime}}$ para $x^{\prime} \sim x$,

$$
\mathbf{P}\left(y \in \mathcal{R}_{x}^{1}\right)=\left[\mathbf{E}\left(p^{T_{x x^{\prime}}}\right)\right]^{n} .
$$

Mas, por condicionamento no primeiro passo do passeio aleatório, concluímos que $\mathbf{E}\left(p^{T_{x x^{\prime}}}\right)$ satisfaz a equação quadrática

$$
\mathbf{E}\left(p^{T_{x x^{\prime}}}\right)=\frac{p}{d+1}\left[1+d\left(\mathbf{E}\left(p^{T_{x x^{\prime}}}\right)\right)^{2}\right] .
$$


Além disso, da continuidade à direita em 0 da função geradora de probabilidade de $T_{x x^{\prime}}$, segue que $\lim _{p \rightarrow 0^{+}} \mathbf{E}\left(p^{T_{x x^{\prime}}}\right)=0$. Então, das duas raízes de (2.3), descarta-se aquela que é ilimitada perto de 0. Assim, fica demonstrada a expressão (2.2) no caso $p<1$.

Finalmente, para $p=1$, utilizamos que a função geradora de probabilidade de $T_{x y}$ é contínua à esquerda em 1 , a fim de obter

$$
\mathbf{P}\left(y \in \mathcal{R}_{x}^{1}\right)=\mathbf{P}\left(T_{x y}<\infty\right)=\lim _{p \rightarrow 1^{-}} \mathbf{E}\left(p^{T_{x y}}\right)=\frac{1}{d^{n}}
$$

Observação 2.2. É interessante notar que, no caso $p=1$, o evento $\left\{y \in \mathcal{R}_{x}^{1}\right\}$ é equivalente ao evento "bancarrota" no conhecido Problema da Ruína do Apostador em que um apostador está jogando contra um adversário infinitamente rico, começa com dist $(x, y)$ unidades monetárias e a cada estágio ou ganha ou perde uma unidade com probabilidades respectivas $d /(d+1)$ e $1 /(d+1)$. Uma explicação detalhada sobre este problema encontra-se, por exemplo, em Feller [6]. Para o caso $p<1$, deve-se considerar que o apostador antes de cada estágio opta com probabilidade $(1-p)$ se deseja interromper o jogo ou não (independentemente do jogo). Por conseguinte, o número total de estágios é uma variável aleatória com distribuição geométrica com parâmetro $(1-p)$.

Observamos ainda que a função geradora de probabilidade de $T_{x y}$ é obtida por meio de um argumento padrão. Uma referência em que também se prova esta fórmula é Woess [18] (na demonstração do lema 1.24, que fornece a função de Green e o raio espectral do passeio aleatório simples na árvore homogênea). 


\subsection{Processos de ramificação dominados por $\operatorname{MS}\left(\mathbb{T}_{d}, p, \eta\right)$}

Completamos nesta seção a prova do teorema 2.1. Recapitulando a idéia, vamos definir uma classe de processos de ramificação dominados pelo modelo dos sapos em $\mathbb{T}_{d}$, para cada um dos quais estabelecemos uma condição suficiente para comportamento supercrítico. Obtemos dessa forma uma seqüência de limitantes superiores para $p_{c}\left(\mathbb{T}_{d}, \eta\right)$, cujo limite é o limitante superior estabelecido no teorema.

Iniciamos com algumas definições.

Definição 2.2. (i) Para $x, y \in \mathcal{V}$, dizemos que $x \leq y$ se $x$ é um dos vértices do caminho entre $\mathbf{0}$ e $y ; x<y$ se $x \leq y$ e $x \neq y$.

(ii) Para $x \neq \mathbf{0}$, seja $\mathbb{T}_{d}^{+}(x)=\{y \in \mathcal{V}: x \leq y\}$; definimos $\mathbb{T}_{d}^{+}(\mathbf{0})=\mathcal{V} \backslash \mathbb{T}_{d}^{+}(z)$, onde $z$ é um vértice vizinho da raiz fixado.

(iii) Para $x \in \mathcal{V}$ e $n \geq 1$, seja $L_{n}(x)=\left\{y \in \mathbb{T}_{d}^{+}(x): \operatorname{dist}(x, y)=n\right\}$.

A figura 2.1 ilustra esses conceitos.

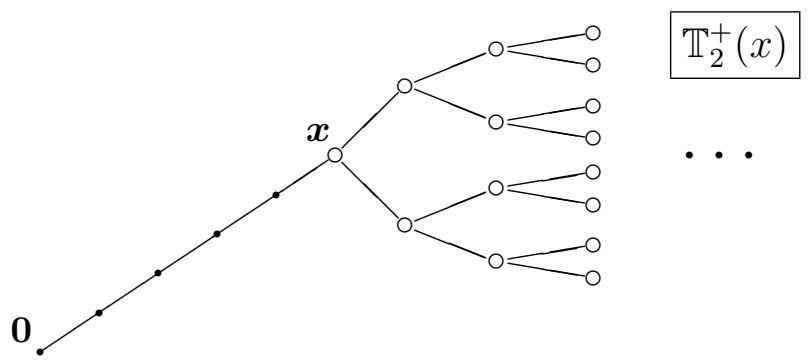

Figura 2.1: Em $\mathbb{T}_{2}$, uma representação do caminho entre a raiz e um vértice $x$ e de $\mathbb{T}_{2}^{+}(x)$. São destacados os vértices no conjunto $\left\{y \in \mathbb{T}_{2}^{+}(x): \operatorname{dist}(x, y) \leq 3\right\}$.

Definição 2.3. Para $x \in \mathcal{V}$ e $y \in L_{n}(x)$, consideremos $x_{0}=x<x_{1}<\cdots<x_{n-1}<$ $x_{n}=y$ o caminho conectando $x$ e $y$. Denotamos por $\{x \stackrel{c}{\rightarrow} y\}$ o evento de que $\{x \rightarrow y\}$ ou de que existam $1 \leq i_{1}<\cdots<i_{k} \leq n-1$ tais que

$$
\left\{x \rightarrow x_{i_{1}}\right\} \bigcap\left(\bigcap_{j=1}^{k-1}\left\{x_{i_{j}} \rightarrow x_{i_{j+1}}\right\}\right) \bigcap\left\{x_{i_{k}} \rightarrow y\right\} .
$$

Além disso, denotamos por $\left\{x_{\stackrel{c}{\hookrightarrow}}^{\nrightarrow} y\right\}$ o complementar deste evento. 
A idéia central para a prova do teorema 2.1 é comparar o modelo dos sapos em $\mathbb{T}_{d}$ ao processo a seguir definido. Fixado $n \geq 1$, para um vértice $x \in \mathcal{V}$, dizemos que $y \in L_{n}(x)$ é descendente de $x$ se o evento $\{x \stackrel{c}{\rightarrow} y\}$ ocorre. O emprego da expressão 'descendente' não é à toa: a evolução das gerações sucessivas a partir da raiz de $\mathbb{T}_{d}$ é governada por um processo de ramificação de Galton-Watson. Além disso, este processo de ramificação é dominado pelo modelo dos sapos, no sentido de que o modelo dos sapos sobrevive caso este processo o faça. Assim, para proceder à prova do limitante superior, vamos obter uma condição suficiente para o comportamento supercrítico do processo de ramificação recém-definido.

A prova prossegue em seis passos, formulados nos lemas 2.2 a 2.6 na seqüência. Antes dos lemas, porém, precisamos de uma definição. Observamos que, nas fórmulas que aparecem daqui em diante, um somatório da forma $\sum_{1}^{0}$ é igual a 0 , e um produto como $\prod_{1}^{0}$ é igual a 1 .

Definição 2.4. (i) Para cada $n \geq 1$, definimos a função $F_{n}$, com domínio $[0,1 / d]$, dada indutivamente por

$$
\begin{aligned}
F_{n}(B)= & {\left[1-\varphi\left(1-B^{n}\right)\right] \prod_{k=1}^{n-1} \varphi\left(1-B^{k}\right) } \\
& +\sum_{j=1}^{n-1}\left[1-\varphi\left(1-B^{n-j}\right)\right] F_{j}(B) \prod_{k=1}^{n-j-1} \varphi\left(1-B^{k}\right) .
\end{aligned}
$$

(ii) Para cada $n \geq 1$, definimos a função $G_{n}$, com domínio $[0,1 / d]$, por

$$
G_{n}(B)=\left(1-p_{0}\right) B^{n}[\gamma(1-B)+\alpha]^{n-1}
$$

Observação 2.3. Lembramos que $p_{0}=\mathbf{P}(\eta=0)<1$ e que $\alpha$ e $\gamma$ são definidas em $(2.1 \mathrm{~b}-\mathrm{c})$. Cumpre também observar que uma notação mais adequada para a função $G_{n}$ seria $G_{n}^{(d)}$, para deixar clara a dependência em $d$. Tendo em mente esta dependência, o sobrescrito é omitido (recorde-se ainda que $d \geq 2$ está fixado). 
No primeiro passo, estudamos $B(p, d)$ como função de $p$, mostrando a monotonicidade e uma importante equivalência.

Lema 2.1. Para todo $d \geq 2$ fixado, $B(p, d)$ é uma função crescente em $p$. Além disso, para $p \in[0,1]$ e $V \in[0,1 / d]$,

$$
B(p, d)=V \Longleftrightarrow p=\frac{(d+1) V}{1+d V^{2}} .
$$

As provas deste e de outros resultados auxiliares à demonstração do teorema 2.1 são apresentadas na subseção 2.3.1 (que se inicia à página 20).

O passo seguinte consiste em estabelecer:

Lema 2.2. Para todo $n \geq 1, \mathbf{P}\left(x_{0} \stackrel{c}{\rightarrow} x_{n}\right)=F_{n}(B(p, d))$.

Desse lema, concluímos que é igual a $d^{n} F_{n}(B(p, d))$ o número esperado de descendentes por indivíduo no processo de ramificação de Galton-Watson introduzido após a definição 2.3. A fim de determinar uma condição suficiente para o comportamento supercrítico deste processo de ramificação (para $n$ grande), usaremos também os próximos resultados:

Lema 2.3. Para todo $n \geq 1$, vale que $F_{n}(B) \geq G_{n}(B)$ para qualquer $B \in[0,1 / d]$.

Lema 2.4. Existe $N=N(d) \geq 1$ tal que, para todo $n \geq N$, a função $G_{n}$ é crescente e existe uma única raiz $\bar{\beta}_{n}=\bar{\beta}_{n}(d)$ da equação $G_{n}(B)=1 / d^{n}$.

Assim, obtemos uma seqüência de limitantes superiores para a probabilidade crítica do modelo dos sapos em $\mathbb{T}_{d}$ :

Lema 2.5. Para quaisquer $d \geq 2$ fixado e $n \geq N$,

$$
p_{c}\left(\mathbb{T}_{d}, \eta\right) \leq \frac{(d+1) \bar{\beta}_{n}}{1+d\left(\bar{\beta}_{n}\right)^{2}} .
$$

Prova. Dado $n \geq 1$, consideremos o processo de ramificação de Galton-Watson imerso em $\operatorname{MS}\left(\mathbb{T}_{d}, p, \eta\right)$ que se inicia com a raiz de $\mathbb{T}_{d}$ e em que $y \in L_{n}(x)$ é descendente de 
um vértice $x$ se o evento $\{x \stackrel{c}{\rightarrow} y\}$ ocorre. Como vimos, este processo de ramificação é dominado pelo modelo dos sapos e tem número médio de descendentes por indivíduo igual a $d^{n} F_{n}(B(p, d))$.

Pelos lemas 2.1, 2.3 e 2.4, concluímos que, para $n \geq N$,

$$
p>\frac{(d+1) \bar{\beta}_{n}}{1+d\left(\bar{\beta}_{n}\right)^{2}} \Rightarrow B(p, d)>\bar{\beta}_{n} \Rightarrow F_{n}(B(p, d)) \geq G_{n}(B(p, d))>\frac{1}{d^{n}} .
$$

Neste caso, o processo de ramificação sobrevive com probabilidade positiva, o mesmo ocorrendo com o modelo dos sapos.

No passo final, enunciamos o seguinte fato.

Lema 2.6. Vale que $\gamma>0$ e

$$
\lim _{n \rightarrow \infty} \bar{\beta}_{n}=\frac{d(\alpha+\gamma)-\sqrt{\Delta}}{2 d \gamma}<\frac{1}{d}
$$

com $\Delta>0$ dado em (2.1d).

Resta-nos, pois, esclarecer como se finaliza a demonstração do teorema 2.1 a partir dos lemas 2.1, 2.5 e 2.6. Como a função $\psi(V)=(d+1) V\left(1+d V^{2}\right)^{-1}$ é contínua em $[0,1 / d]$, fazendo $n \rightarrow \infty$ em (2.5), obtemos que

$$
\frac{(d+1) \bar{\beta}}{1+d(\bar{\beta})^{2}}
$$

é um limitante superior para $p_{c}\left(\mathbb{T}_{d}, \eta\right)$, onde $\bar{\beta}=\bar{\beta}(d)$ é o limite da seqüência $\left\{\bar{\beta}_{n}\right\}_{n \geq N}$ dado em (2.6). A fórmula para o limitante superior apresentada no teorema 2.1 é obtida ao notarmos que

$$
\begin{aligned}
1+d(\bar{\beta})^{2} & =1+\frac{[d(\alpha+\gamma)-\sqrt{\Delta}]^{2}}{4 d \gamma^{2}} \\
& =\frac{4 d \gamma^{2}+2 d^{2}(\alpha+\gamma)^{2}-2 d(\alpha+\gamma) \sqrt{\Delta}-4 d \gamma}{4 d \gamma^{2}} \\
& =\frac{(\alpha+\gamma)[d(\alpha+\gamma)-\sqrt{\Delta}]-2 \gamma(1-\gamma)}{2 \gamma^{2}} .
\end{aligned}
$$

Por fim, o limitante superior é estritamente menor que 1 , já que $\bar{\beta}<1 / d$. 


\subsubsection{Provas dos lemas}

Prova do lema 2.1. Recordamos da prova da proposição 2.1 que $B(p, d)$ é a função geradora de probabilidade de $T_{x x^{\prime}}$, onde $T_{x x^{\prime}}$ denota o primeiro tempo em que um passeio aleatório simples em $\mathbb{T}_{d}$ começando em um vértice $x$ visita um vértice $x^{\prime} \sim x$ fixado. Como $\mathbf{P}\left(1 \leq T_{x x^{\prime}}<\infty\right)>0$, temos que $B(p, d)$ é crescente em $p$. Assim, concluímos que $B(p, d)$ como função de $p$ possui uma inversa. A equivalência (2.4) decorre, portanto, da expressão (2.3).

Prova do lema 2.2. Seja $n \geq 2$ e, para cada $1 \leq j \leq n-1$, consideremos o evento $\left\{x_{0} \dashv x_{j}\right\}=\left\{x_{0} \rightarrow x_{j}, x_{0} \nrightarrow x_{j+1}\right\}$. Notamos que

$$
\mathbf{P}\left(x_{0} \stackrel{c}{\rightarrow} x_{n}\right)=\mathbf{P}\left(x_{0} \rightarrow x_{n}\right)+\sum_{j=1}^{n-1} \mathbf{P}\left(x_{0} \dashv x_{j}\right) \mathbf{P}\left(\bigcup_{k=1}^{j}\left\{x_{k} \stackrel{c}{\rightarrow} x_{n}\right\}\right),
$$

$\operatorname{com} \mathbf{P}\left(x_{0} \dashv x_{j}\right)=\mathbf{P}\left(x_{0} \rightarrow x_{j}\right)-\mathbf{P}\left(x_{0} \rightarrow x_{j+1}\right)$.

Portanto, colocando em evidência os fatores comuns, obtemos

$$
\begin{aligned}
\mathbf{P}\left(x_{0} \stackrel{c}{\rightarrow} x_{n}\right)= & \mathbf{P}\left(x_{0} \rightarrow x_{n}\right) \mathbf{P}\left(x_{1} \stackrel{c}{\rightarrow} x_{n}, \ldots, x_{n-1} \stackrel{c}{\hookrightarrow} x_{n}\right) \\
& +\sum_{j=1}^{n-2} \mathbf{P}\left(x_{0} \rightarrow x_{n-j}\right) \mathbf{P}\left(x_{1} \stackrel{c}{\rightarrow} x_{n}, \ldots, x_{n-j-1} \stackrel{c}{\rightarrow} x_{n}, x_{n-j} \stackrel{c}{\rightarrow} x_{n}\right) \\
& +\mathbf{P}\left(x_{0} \rightarrow x_{1}\right) \mathbf{P}\left(x_{1} \stackrel{c}{\rightarrow} x_{n}\right) \\
= & \mathbf{P}\left(x_{0} \rightarrow x_{n}\right) \mathbf{P}\left(x_{1} \nrightarrow x_{n}, \ldots, x_{n-1} \nrightarrow x_{n}\right) \\
& +\sum_{j=1}^{n-2} \mathbf{P}\left(x_{0} \rightarrow x_{n-j}\right) \mathbf{P}\left(x_{1} \nrightarrow x_{n-j}, \ldots, x_{n-j-1} \nrightarrow x_{n-j}, x_{n-j} \stackrel{c}{\rightarrow} x_{n}\right) \\
& +\mathbf{P}\left(x_{0} \rightarrow x_{1}\right) \mathbf{P}\left(x_{1} \stackrel{c}{\rightarrow} x_{n}\right) .
\end{aligned}
$$

Usando a proposição 2.1, o resultado segue por indução em $n$.

Visando às demonstrações dos lemas 2.3, 2.4 e 2.6, estabelecemos o próximo resultado.

Lema 2.7. (a) Para $1-\frac{1}{d} \leq s \leq 1$,

$$
\gamma s+p_{0} \leq \varphi(s) \leq 1-\left(\alpha-p_{0}\right)(1-s) .
$$

(b) Temos que $\theta<1, \alpha \geq 1$ e $\gamma>0$. 
Prova. (a) Para $1-\frac{1}{d} \leq s \leq 1$,

$$
\gamma s+p_{0}=p_{0}+s \sum_{j=1}^{\infty} p_{j}\left(1-\frac{1}{d}\right)^{j-1} \leq \varphi(s),
$$

e, visto que $\varphi$ é convexa,

$$
\varphi(s) \leq 1+d(1-\theta)(s-1)=1-\left(\alpha-p_{0}\right)(1-s)
$$

(b) Também da convexidade de $\varphi$, segue que $\varphi(s) \leq 1+\left(1-p_{0}\right)(s-1)$ para todo $s \in[0,1]$; fazendo $s=1-1 / d$, concluímos que $\alpha \geq 1$. Como $p_{0}<1$, temos que $\varphi$ é crescente, $\log 0 \quad \theta<1$ e $\gamma>0$.

Prova do lema 2.3. Para cada $n \geq 1$, consideremos a função $H_{n}$, com domínio $[0,1 / d]$, definida indutivamente por

$$
\begin{aligned}
H_{n}(B)=\left(\alpha-p_{0}\right)\{ & B^{n}\left[\gamma(1-B)+p_{0}\right]^{n-1} \\
& \left.+\sum_{j=1}^{n-1} B^{n-j} H_{j}(B)\left[\gamma(1-B)+p_{0}\right]^{n-j-1}\right\} .
\end{aligned}
$$

Tendo em vista a definição de $F_{n}$ e usando o lema 2.7, vamos mostrar que, para todo $n \geq 1, F_{n}(B) \geq H_{n}(B)$ para qualquer $B \in[0,1 / d]$. Para isso, utilizamos indução em $n$. O caso inicial $n=1$ é conseqüência direta da segunda desigualdade em (2.7). Se, para todo $j<n, F_{j}(B) \geq H_{j}(B)$ para qualquer $B$, então

$$
\begin{aligned}
F_{n}(B) \geq & \left(\alpha-p_{0}\right) B^{n}\left[\gamma(1-B)+p_{0}\right]^{n-1} \\
& +\sum_{j=1}^{n-1}\left(\alpha-p_{0}\right) B^{n-j} F_{j}(B)\left[\gamma(1-B)+p_{0}\right]^{n-j-1} \geq H_{n}(B),
\end{aligned}
$$

o que completa a indução.

Notamos que, para $n \geq 1$,

$$
\begin{aligned}
H_{n+1}(B) & =B\left[\gamma(1-B)+p_{0}\right] H_{n}(B)+\left(\alpha-p_{0}\right) B H_{n}(B) \\
& =B[\gamma(1-B)+\alpha] H_{n}(B)
\end{aligned}
$$

portanto $H_{n}(B)=\left(\alpha-p_{0}\right) B^{n}[\gamma(1-B)+\alpha]^{n-1}, n \geq 1$. Lembrando que $\alpha \geq 1$ e $\gamma>0$, o resultado segue. 
Prova do lema 2.4. Mostramos primeiramente que, para todo $n \geq 1, G_{n}$ é uma função crescente. Para isso, observamos que, para cada $n \geq 1, G_{n}$ é contínua e

$$
\frac{d G_{n}(B)}{d B}=n\left(1-p_{0}\right) B^{n-1}[\gamma(1-B)+\alpha]^{n-2}\left[\gamma\left(1-2 B+\frac{B}{n}\right)+\alpha\right]
$$

que é estritamente positiva para todo $B \in(0,1 / d)$.

Assim, o resultado é obtido ao se notar que $G_{n}(0)=0$ e que

$$
G_{n}(1 / d)=\left(1-p_{0}\right)\left(\frac{1}{d^{n}}\right)\left[\gamma\left(1-\frac{1}{d}\right)+\alpha\right]^{n-1} \geq \frac{1}{d^{n}}
$$

para $n$ suficientemente grande.

Prova do lema 2.6* . Já mostramos que $\gamma>0$. Afirmamos que a seqüência $\left\{\bar{\beta}_{n}\right\}_{n \geq N}$ é não-crescente. De fato, para todo $n \geq N$,

$$
\begin{aligned}
d^{n+1} G_{n+1}\left(\bar{\beta}_{n}\right) & =d^{n+1} \bar{\beta}_{n}\left[\gamma\left(1-\bar{\beta}_{n}\right)+\alpha\right] G_{n}\left(\bar{\beta}_{n}\right) \\
& =d \bar{\beta}_{n}\left[\gamma\left(1-\bar{\beta}_{n}\right)+\alpha\right] \\
& \geq d\left(1-p_{0}\right)^{1 / n} \bar{\beta}_{n}\left[\gamma\left(1-\bar{\beta}_{n}\right)+\alpha\right]^{1-\frac{1}{n}}=1,
\end{aligned}
$$

portanto $\bar{\beta}_{n+1} \leq \bar{\beta}_{n}$.

A seguir, observamos que a seqüência de funções $\left\{G_{n}(B)^{1 / n}\right\}_{n \geq N}$ converge uniformemente quando $n \rightarrow \infty$ para a função $G(B)=B[\gamma(1-B)+\alpha]$. Por conseguinte, definindo $\bar{\beta}=\lim _{n \rightarrow \infty} \bar{\beta}_{n}$, concluímos que $G(\bar{\beta})=1 / d$. Daí, segue que $\bar{\beta} \in[0,1 / d]$ é uma raiz do polinômio de grau 2 em $\beta$

$$
\phi(\beta)=d \gamma \beta^{2}-d(\alpha+\gamma) \beta+1
$$

Como $d \gamma>0, \phi(0)=1$ e $\phi(1 / d)=(1-d)(1-\theta)<0$, temos que $\phi$ possui duas raízes reais distintas, uma no intervalo $(0,1 / d)$ e a outra maior que $1 / d$. Então, $\bar{\beta} \in(0,1 / d)$ é a menor das raízes de $\phi$, e o resultado está provado.

*Para uma explicação mais detalhada da parte relacionada à convergência uniforme, consulte-se o apêndice (p. 30). 


\subsection{Observações}

Observação 2.4. Considerando o caso de configuração inicial de uma partícula por vértice, uma passagem importante na prova do limitante superior é a comparação

$$
F_{n}(B(p, d)) \geq G_{n}(B(p, d))=(B(p, d))^{n}(2-B(p, d))^{n-1} .
$$

A fim de fornecer uma interpretação probabilística para esta desigualdade, definimos, para $x \in \mathcal{V}$ e $y \in L_{n}(x)$, o evento $\{x \stackrel{e}{\rightarrow} y\}$. Esta definição será indutiva em função da distância entre os dois vértices. Seja $x_{0}=x<x_{1}<\cdots<x_{n-1}<x_{n}=y$ o caminho conectando $x$ e $y$. Se $n=1$, fazemos $\left\{x_{0} \stackrel{e}{\rightarrow} x_{1}\right\}=\left\{x_{0} \rightarrow x_{1}\right\}$ e, se $n \geq 2$, definimos

$$
\left\{x_{0} \stackrel{e}{\rightarrow} x_{n}\right\}=\left\{x_{0} \rightarrow x_{n}\right\} \bigcup\left(\bigcup_{j=1}^{n-1}\left\{x_{0} \dashv x_{j}\right\} \cap\left\{x_{j} \stackrel{e}{\rightarrow} x_{n}\right\}\right) .
$$

Recorde-se que $\left\{x_{0} \dashv x_{j}\right\}=\left\{x_{0} \rightarrow x_{j}, x_{0} \nrightarrow x_{j+1}\right\}, 1 \leq j \leq n-1$.

Observamos então que $\left\{x_{0} \stackrel{c}{\rightarrow} x_{n}\right\} \supset\left\{x_{0} \stackrel{e}{\rightarrow} x_{n}\right\}$, logo

$$
\mathbf{P}\left(x_{0} \stackrel{c}{\rightarrow} x_{n}\right) \geq \mathbf{P}\left(x_{0} \stackrel{e}{\rightarrow} x_{n}\right) .
$$

Além disso, notamos que as funções $G_{n}(B)=B^{n}(2-B)^{n-1}, n \geq 1$, poderiam ter sido definidas indutivamente por

$$
G_{n}(B)=B^{n}+\sum_{j=1}^{n-1}\left(B^{j}-B^{j+1}\right) G_{n-j}(B), n \geq 1 .
$$

De fato, definindo as funções $G_{n}$ como em $(2.9)$, temos que $G_{1}(B)=B$ e $G_{n+1}(B)=$ $B(2-B) G_{n}(B)$ para $n \geq 1$. Da definição de $\left\{x_{0} \stackrel{e}{\rightarrow} x_{n}\right\}$, segue que

$$
\mathbf{P}\left(x_{0} \stackrel{e}{\rightarrow} x_{n}\right)=G_{n}(B(p, d)) .
$$

Assim, fica estabelecida uma interpretação probabilística para a comparação (2.8).

Observação 2.5. Reiteramos que em Lebensztayn et al. [9] o caso $\eta \equiv 1$ é abordado separadamente. Nesse caso, as funções $F_{n}$ são polinomiais e uma seqüência de limitantes superiores para a probabilidade crítica $p_{c}\left(\mathbb{T}_{d}\right)$ pode ser obtida em função das raízes 
das equações $F_{n}(B)=1 / d^{n}, n \geq 1$. No entanto, conforme se discute na seção 4 do artigo, ainda assim há a necessidade de definir funções $G_{n}$ tais que $F_{n}(B) \geq G_{n}(B)$ para todo $B$. Esta necessidade surge em razão das dificuldades técnicas em trabalhar com as funções $F_{n}$ no que tange a demonstrar a convergência da seqüência de limitantes superiores. Quanto à escolha das funções $G_{n}$, como se observa no artigo, simplicidade e clareza foram os fatores determinantes para a escolha efetuada.

Incluída esta explicação, justifica-se a opção feita na tese por tratar diretamente do caso de configuração inicial aleatória. 


\section{Capítulo 3}

\section{Correção assintótica}

Visamos neste capítulo a mostrar o valor assintótico do limitante superior para a probabilidade crítica estabelecido no teorema 2.1. Antes disso, apresentamos um limitante inferior para a probabilidade crítica. Usando então o teorema do confronto do Cálculo Diferencial e Integral, obteremos o valor assintótico de $p_{c}\left(\mathbb{T}_{d}, \eta\right)$. Provaremos, assim, que o limitante superior dado no teorema 2.1 é assintoticamente correto, ou seja, converge quando $d \rightarrow \infty$ para o valor assintótico da probabilidade crítica.

\subsection{Limitante inferior para a probabilidade crítica}

Lembramos que $m=\mathbf{E} \eta$ é o número inicial esperado de partículas por vértice.

Proposição 3.1. Suponhamos que $m<\infty$. Então, para todo $d \geq 2$ fixado,

$$
p_{c}\left(\mathbb{T}_{d}, \eta\right) \geq \frac{d+1}{d(m+1)+1}
$$

Prova. Baseia-se no seguinte fato: dentre os vértices vizinhos a uma partícula ativa no instante $n \geq 1$, existe pelo menos um, tal que o salto para ele não implica a ativação de novas partículas. Seja $Y_{n}$ o número de partículas ativas no instante $n$ em $\operatorname{MS}\left(\mathbb{T}_{d}, p, \eta\right)$. Pelo fato anterior, dado que $\left\{Y_{n}=k\right\}$ para um $n \geq 1$ fixado, temos que $Y_{n+1}$ não é maior que uma soma de $k$ variáveis aleatórias, cada uma assumindo os valores 0,1 e $j \geq 2$ com probabilidades respectivas $(1-p), \frac{p\left(1+d p_{0}\right)}{d+1}$ e $\frac{p d p_{j-1}}{d+1}$. 
Por conseguinte, para $n \geq 1$,

$$
\mathbf{E}\left(Y_{n+1} \mid Y_{n}=k\right) \leq\left[\frac{d(m+1)+1}{d+1}\right] p k,
$$

$\log 0$

$$
\mathbf{E}\left(Y_{n+1}\right) \leq\left[\frac{d(m+1)+1}{d+1}\right] p \mathbf{E}\left(Y_{n}\right)
$$

Assim, se $p<(d+1)[d(m+1)+1]^{-1}$, temos que $\lim _{n \rightarrow \infty} \mathbf{E}\left(Y_{n}\right)=0$.

Observação 3.1. A proposição 3.1 é um caso particular da proposição 1.2 de Alves et al. [2], que apresenta, sob a hipótese de que $\mathbf{E} \eta<\infty$, um limitante inferior para a probabilidade crítica do modelo dos sapos em um grafo de grau limitado. A prova no artigo é baseada no mesmo fato, mas se utiliza uma comparação do modelo dos sapos a um processo de ramificação de Galton-Watson. Optamos na tese por uma demonstração alternativa, usando um argumento de supermartingal. 


\subsection{Valor assintótico}

Nosso objetivo principal neste capítulo é estabelecer o seguinte resultado, cuja prova é apresentada na seção 3.3.

Teorema 3.1. O limitante superior para $p_{c}\left(\mathbb{T}_{d}, \eta\right)$ dado no teorema 2.1 converge quando $d \rightarrow \infty$ para

$$
\left\{\begin{array}{ccc}
(1+m)^{-1} & \text { se } & m<\infty \\
0 & \text { se } & m=\infty
\end{array}\right.
$$

Usando o teorema do confronto, a proposição 3.1 e o teorema 3.1, obtemos o valor assintótico da probabilidade crítica do modelo dos sapos em $\mathbb{T}_{d}$ :

Teorema 3.2. Temos que

$$
\lim _{d \rightarrow \infty} p_{c}\left(\mathbb{T}_{d}, \eta\right)=\left\{\begin{array}{ccc}
(1+m)^{-1} & \text { se } & m<\infty \\
0 & \text { se } & m=\infty
\end{array}\right.
$$

Assim, fica estabelecida a correção assintótica do limitante superior dado no teorema 2.1.

Observação 3.2. Uma prova probabilística do caso $m<\infty$ do teorema 3.2 encontra-se em Alves et al. [2] (teorema 1.7). 


\subsection{Prova do teorema 3.1}

Primeiramente, devemos considerar

$$
\theta_{d}=\varphi(1-1 / d), \alpha_{d}=p_{0}+d\left(1-\theta_{d}\right) \text { e } \gamma_{d}=\frac{d}{d-1}\left(\theta_{d}-p_{0}\right)
$$

como funções de $d$, o que indicamos por meio do subscrito. Para demonstrar o teorema 3.1, usaremos o seguinte resultado.

Lema 3.1. Temos que

(a) $\alpha_{d}$ e $\gamma_{d}$ são não-decrescentes em d.

(b) $\lim _{d \rightarrow \infty} \gamma_{d}=1-p_{0} \quad$ e $\lim _{d \rightarrow \infty} \alpha_{d}=\left\{\begin{array}{ccc}p_{0}+m & \text { se } & m<\infty \\ \infty & \text { se } & m=\infty\end{array}\right.$

Prova. (a) A afirmação relativa a $\gamma_{d}$ é verdadeira, pois

$$
\gamma_{d}=\sum_{j=1}^{\infty} p_{j}\left(1-\frac{1}{d}\right)^{j-1}
$$

Com respeito a $\alpha_{d}$, notamos que, se $p_{0}+p_{1}=1$, então $\alpha_{d}=1$ para todo $d \geq 2$. Por outro lado, se $p_{0}+p_{1}<1$, temos que $\varphi$ é estritamente convexa, logo

$$
\varphi(s)>\theta_{d}+\varphi^{\prime}(1-1 / d)\left[s-\left(1-\frac{1}{d}\right)\right] \text { para } s \neq 1-\frac{1}{d}
$$

onde $\varphi^{\prime}$ denota a derivada de $\varphi$. Fazendo $s=1$ na última desigualdade, concluímos que a derivada de $\alpha_{d}$ em relação a $d$ é estritamente positiva. Portanto, $\alpha_{d}$ é crescente em $d$ se $p_{0}+p_{1}<1$.

(b) Decorrem, respectivamente, dos seguintes fatos:

$$
\lim _{s \rightarrow 1^{-}} \varphi(s)=\varphi(1)=1 \quad \text { e } \quad \lim _{s \rightarrow 1^{-}} \frac{\varphi(s)-1}{s-1}=\lim _{s \rightarrow 1^{-}} \varphi^{\prime}(s)=m .
$$

Convém então recordarmos a passagem final da demonstração do teorema 2.1. Para cada $d \geq 2$, o limitante superior para $p_{c}\left(\mathbb{T}_{d}, \eta\right)$ neste teorema é dado por

$$
\frac{(d+1) \bar{\beta}_{d}}{1+d\left(\bar{\beta}_{d}\right)^{2}}
$$


onde $\bar{\beta}_{d}$ é a única raiz no intervalo $(0,1 / d)$ do polinômio em $\beta$

$$
f_{d}(\beta)=\frac{d \gamma_{d}}{\alpha_{d}+\gamma_{d}} \beta^{2}-d \beta+\frac{1}{\alpha_{d}+\gamma_{d}}
$$

Assim, definindo $\bar{\lambda}_{d}=d \bar{\beta}_{d}$, temos que $\bar{\lambda}_{d}$ é a única raiz em $(0,1)$ do polinômio em $\lambda$

$$
g_{d}(\lambda)=\frac{\gamma_{d}}{d\left(\alpha_{d}+\gamma_{d}\right)} \lambda^{2}-\lambda+\frac{1}{\alpha_{d}+\gamma_{d}}
$$

Ademais, o teorema 3.1 estará provado uma vez que mostrarmos

$$
\lim _{d \rightarrow \infty} \bar{\lambda}_{d}=\left\{\begin{array}{ccc}
(1+m)^{-1} & \text { se } & m<\infty \\
0 & \text { se } & m=\infty
\end{array}\right.
$$

Prova de (3.1). Consideremos o caso $m<\infty$. Observamos que, para cada $d \geq 2$, o polinômio quadrático $g_{d}$ é decrescente em $[0,1]$, pois seu ponto de mínimo é

$$
\frac{d\left(\alpha_{d}+\gamma_{d}\right)}{2 \gamma_{d}}>1
$$

Notamos a seguir que, pelo lema 3.1 (a), a função

$$
h_{d}(\lambda)=\left(\alpha_{d}+\gamma_{d}\right) g_{d}(\lambda)=-\lambda\left[\alpha_{d}+\gamma_{d}\left(1-\frac{\lambda}{d}\right)\right]+1
$$

satisfaz $h_{d}(\lambda) \geq h_{d+1}(\lambda)$ para todo $\lambda \in[0,1]$. Então,

$$
g_{d}\left(\bar{\lambda}_{d+1}\right)=\left(\frac{1}{\alpha_{d}+\gamma_{d}}\right) h_{d}\left(\bar{\lambda}_{d+1}\right) \geq\left(\frac{1}{\alpha_{d}+\gamma_{d}}\right) h_{d+1}\left(\bar{\lambda}_{d+1}\right)=0 .
$$

Daí, segue que $\bar{\lambda}_{d+1} \leq \bar{\lambda}_{d}$ para todo $d \geq 2$, logo existe $\lambda^{*}=\lim _{d \rightarrow \infty} \bar{\lambda}_{d}$.

Do lema 3.1 (b), concluímos que a seqüência $\left\{g_{d}\right\}_{d \geq 2}$ converge pontualmente em $[0,1]$, quando $d \rightarrow \infty$, para a função

$$
g(\lambda)=-\lambda+\frac{1}{1+m}
$$

Como esta convergência é uniforme, temos que $g\left(\lambda^{*}\right)=0$, portanto $\lambda^{*}=(1+m)^{-1}$.

O caso $m=\infty$ é análogo $(\operatorname{com} g(\lambda)=-\lambda)$. 


\section{Apêndice}

\section{Convergência uniforme}

Ao final deste apêndice, apresentamos um resultado relacionado à convergência uniforme que é essencial em duas passagens da tese: nas provas do limitante superior para a probabilidade crítica (lema 2.6) e do valor assintótico (expressão (3.1)). Antes, porém, recordamos os conceitos de convergência pontual e convergência uniforme e enunciamos uma condição suficiente para convergência uniforme, a qual é utilizada nos dois contextos mencionados. Citamos Lima [12] (Capítulo X) para o leitor interessado em mais detalhes sobre o assunto, muito embora ambos os teoremas a seguir constem neste livro como exercícios. Exatamente a dificuldade em encontrar as demonstrações é o motivo pelo qual optamos por apresentá-las.

Definição A.1. Seja $\left\{f_{n}\right\}_{n \geq 1}$ uma seqüência de funções a valores reais definidas em $X \subset \mathbb{R}$.

(i) Dizemos que a seqüência $\left\{f_{n}\right\}_{n \geq 1}$ converge pontualmente (ou simplesmente) para a função $f: X \rightarrow \mathbb{R}$ quando, para cada $x \in X$, a seqüência de números $\left\{f_{n}(x)\right\}_{n \geq 1}$ converge para o número $f(x)$.

(ii) Dizemos que a seqüência $\left\{f_{n}\right\}_{n \geq 1}$ converge uniformemente para a função $f: X \rightarrow \mathbb{R}$ quando, para todo $\varepsilon>0$ dado, existe $N \in \mathbb{N}$ tal que $n \geq N$ implica que

$$
\left|f_{n}(x)-f(x)\right|<\varepsilon,
$$

seja qual for $x \in X$. 
Em cada caso, dizemos que $\left\{f_{n}\right\}$ converge pontualmente/uniformemente para $f$ em $X$.

O próximo resultado estabelece uma condição suficiente para convergência uniforme, sendo particularmente útil nos dois contextos considerados.

Teorema A.1. Suponhamos que $\left\{f_{n}\right\}$ é uma seqüência de funções monótonas a valores reais, definidas em $[a, b]$. Se $\left\{f_{n}\right\}$ converge pontualmente para uma função contínua $f$ em $[a, b]$, então $\left\{f_{n}\right\}$ converge uniformemente para $f$ em $[a, b]$.

Prova. Temos que $f$ é uniformemente contínua, pois é uma função contínua definida em um conjunto compacto (Lima [12], Teorema 17, Capítulo VII). Assim, dado $\varepsilon>0$, existe $\delta>0$ tal que $|f(x)-f(y)|<\varepsilon / 5$ sempre que $|x-y|<\delta$. Fixamos um número finito de pontos $a=x_{0}<x_{1}<\cdots<x_{k}=b$ com $x_{i}-x_{i-1}<\delta$ para todo $1 \leq i \leq k$. Como $\left\{f_{n}\right\}$ converge pontualmente para $f$, podemos tomar um inteiro $N$ tal que $\left|f_{n}\left(x_{i}\right)-f\left(x_{i}\right)\right|<\varepsilon / 5$ para cada $0 \leq i \leq k$ e todo $n \geq N$.

Consideremos $n \geq N$ e suponhamos que $f_{n}$ é não-crescente. Se $x \in[a, b]$, então $x_{i-1} \leq x \leq x_{i}$ para algum $1 \leq i \leq k, \operatorname{logo}$

$$
\begin{aligned}
\mid f_{n}(x) & -f_{n}\left(x_{i}\right) \mid=f_{n}(x)-f_{n}\left(x_{i}\right) \leq f_{n}\left(x_{i-1}\right)-f_{n}\left(x_{i}\right) \\
& =\left[f_{n}\left(x_{i-1}\right)-f\left(x_{i-1}\right)\right]+\left[f\left(x_{i-1}\right)-f\left(x_{i}\right)\right]+\left[f\left(x_{i}\right)-f_{n}\left(x_{i}\right)\right]<3 \varepsilon / 5 .
\end{aligned}
$$

Uma desigualdade semelhante vale se $f_{n}$ é não-decrescente. Portanto,

$$
\begin{aligned}
\left|f_{n}(x)-f(x)\right| & \leq\left|f_{n}(x)-f_{n}\left(x_{i}\right)\right|+\left|f_{n}\left(x_{i}\right)-f\left(x_{i}\right)\right|+\left|f\left(x_{i}\right)-f(x)\right| \\
& <3 \varepsilon / 5+\varepsilon / 5+\varepsilon / 5=\varepsilon,
\end{aligned}
$$

para todo $x \in[a, b]$ e todo $n \geq N$. Isto mostra que $\left\{f_{n}\right\}$ converge uniformemente para $f$ em $[a, b]$. 
Eis o resultado a que nos referimos no início do apêndice:

Teorema A.2. Seja $\left\{f_{n}\right\}$ uma seqüência de funções a valores reais, definidas em $X \subset$ $\mathbb{R}$, que converge uniformemente para uma função contínua $f$ em $X$. Se $\lim _{n \rightarrow \infty} x_{n}=x$, onde $x \in X$ e cada $x_{n} \in X$, então $\lim _{n \rightarrow \infty} f_{n}\left(x_{n}\right)=f(x)$.

Prova. Dado $\varepsilon>0$, pela convergência uniforme, existe $N_{1} \in \mathbb{N}$ tal que $\mid f_{n}(y)-$ $f(y) \mid<\varepsilon / 2$ para todo $n \geq N_{1}$ e todo $y \in X$. Como $f$ é contínua em $x$, temos que $\lim _{n \rightarrow \infty} f\left(x_{n}\right)=f(x), \operatorname{logo}$ existe $N_{2} \in \mathbb{N}$ tal que $\left|f\left(x_{n}\right)-f(x)\right|<\varepsilon / 2$ para todo $n \geq N_{2}$. Fazendo $N=$ máx $\left\{N_{1}, N_{2}\right\}$, obtemos que

$$
\left|f_{n}\left(x_{n}\right)-f(x)\right| \leq\left|f_{n}\left(x_{n}\right)-f\left(x_{n}\right)\right|+\left|f\left(x_{n}\right)-f(x)\right|<\varepsilon
$$

para todo $n \geq N$, portanto $\lim _{n \rightarrow \infty} f_{n}\left(x_{n}\right)=f(x)$. 


\section{Referências Bibliográficas}

[1] ALVES, O. S. M. Transição de fase e forma assintótica em um modelo de reação em cadeia. Tese (Doutorado em Estatística) - Instituto de Matemática e Estatística, Universidade de São Paulo, São Paulo, 2001.

[2] AlveS, O. S. M.; MACHADO, F. P.; POPOV, S. Yu. Phase transition for the frog model. Electron. J. Probab., v. 7, n. 16, 21 p., 2002.

[3] AlveS, O. S. M.; MACHADO, F. P.; POPOV, S. Yu. The shape theorem for the frog model. Ann. Appl. Probab., v. 12, n. 2, p. 533-546, 2002.

[4] Alves, O. S. M.; MACHAdO, F. P.; POPOV, S. Yu.; RAVISHANKAR, K. The shape theorem for the frog model with random initial configuration. Markov Process. Related Fields, v. 7, n. 4, p. 525-539, 2001.

[5] BOLLOBÁS, B. Modern graph theory. New York: Springer-Verlag, 1998.

[6] FELLER, W. An introduction to probability theory and its applications, Volume I. 3rd. ed. New York: John Wiley \& Sons, 1968.

[7] FONTES, L. R.; MACHADO, F. P.; SARKAR, A. The critical probability for the frog model is not a monotonic function of the graph. J. Appl. Probab., v. 41, n. 1, p. $292-298,2004$.

[8] GRIMMETT, G. Percolation. 2nd. ed. Berlin: Springer-Verlag, 1999.

[9] LEBEnSZTAYN, E.; MACHADO, F. P.; POPOV, S. An improved upper bound for the critical probability of the frog model on homogeneous trees. J. Statist. Phys., v. 119, n. 1-2, p. 331-345, 2005.

[10] LEICHSENRING, A. R. Não monotonicidade do parâmetro crítico no modelo dos sapos. Dissertação (Mestrado em Estatística) - Instituto de Matemática e Estatística, Universidade de São Paulo, São Paulo, 2003. 
[11] LIGGETT, T. M. Stochastic interacting systems: contact, voter and exclusion processes. Berlin: Springer-Verlag, 1999.

[12] LIMA, E. L. Curso de análise, Volume 1. 11. ed. Rio de Janeiro: Associação Instituto Nacional de Matemática Pura e Aplicada, 2004. (Projeto Euclides).

[13] LYONS, R.; PERES, Y. Probability on trees and networks. Disponível em: $<$ http://mypage.iu.edu/rrdlyons/prbtree/prbtree.html>. Acesso em: 4 jun. 2005. Livro em preparação, a ser publicado pela Cambridge University Press.

[14] POPOV, S. Yu. Frogs and some other interacting random walks models. In: DISCRETE RANDOM WALKS, DRW'03, 2003, Paris. Discrete Mathematics and Theoretical Computer Science Proceedings. Paris: DMTCS, 2003. v. AC, p. 277288.

[15] POPOV, S. Yu. Frogs in random environment. J. Statist. Phys., v. 102, n. 1-2, p. 191-201, 2001.

[16] RAMÍREZ, A. F.; SIDORAVICIUS, V. Asymptotic behavior of a stochastic combustion growth process. J. Eur. Math. Soc., v. 6, n. 3, p. 293-334, 2004.

[17] TELCS, A.; WORMALD, N. C. Branching and tree indexed random walks on fractals. J. Appl. Probab., v. 36, n. 4, p. 999-1011, 1999.

[18] WOESS, W. Random walks on infinite graphs and groups. Cambridge: Cambridge University Press, 2000. 